\title{
6 Early prediction of breast cancer from mammogram images using classification methods: a comparison
}

\begin{abstract}
Today the deaths of women in the age group 15-54 are increasing due to malignant cells in breast. It is recognized as the main cause for deaths of women. Day by day, the number of patients are increasing, because its important factors have not been identified yet, and it is unable to prevent. Therefore, the possibility of improvement is only the early diagnosis. Machine learning (ML) techniques can assist the physicians by expanding tools for detection at initial stage and analysis of breast cancer, thus increasing the probability of patient's survival. For routine breast screening, currently mammography is the commonly accepted imaging method. First, aim of this survey is to develop techniques that are helpful for the prior detection of cancer using different classification methods such as support vector machine, decision tree, artificial neural network (NN), logistic regression and ML-NN, and a comparative study of extracting the feature with and without removing pectoral muscle in the initial stage [1] using a different and effectual method is the another goal behind this study.
\end{abstract}

Keywords: Breast cancer, data analysis, machine learning, classification, prediction

\subsection{Introduction}

Nowadays, women's death has been increasing because of the breast cancer (BC). The abnormal growth of the cell is the main reason of the BC. Generally, identifying the cancer specifically in middle-aged women is quite difficult. It cannot be totally prevented, because the main reason behind this is not known till yet. To decrease the death rate, there is a need to identify $\mathrm{BC}$ in the early stage and the better treatment must be an available option to patients. If $\mathrm{BC}$ is identified in the earlier stage, number of patients suffering from this disease will be decreased. Mammography enables to detect cancer in its initial stage. It cannot eliminate the cancer, but can protect the life of cancer patients. So, they help to detect intangible tumors and increase the durability rate. In biological and medical field, preprocessing is the very important concept.

It is used for diagnosing abnormal cases, image analyzing and extraction of useful information. 
This mammogram image is examined by radiologists (Figure 6.1). It detects the abnormalities. It can also classify whether the cancer is in benign or malignant category. The radiologist predicts unsuccessful result at many times to identify the false negative (FN) and false positive (FP). Therefore, it is impossible to detect the correct abnormality by a human radiologist. From digitized mammogram, automatically detect the suspicious lesion; for increasing the quality of the image some preprocessing steps have been done. Better quality image can be obtained by eliminating unwanted areas in the background of the mammogram image. Two main preprocessing steps are the removal of pectoral muscles and noise. The main goal of eliminating the noise from image is to develop noise-free data for further preprocessing. Pectoral muscle is another important thing, as it interrupts the accuracy for identification of bosom malignant growth from the mammogram picture. Hence, extraction of pectoral muscles stage performs a significant part in successful identification of cancer.

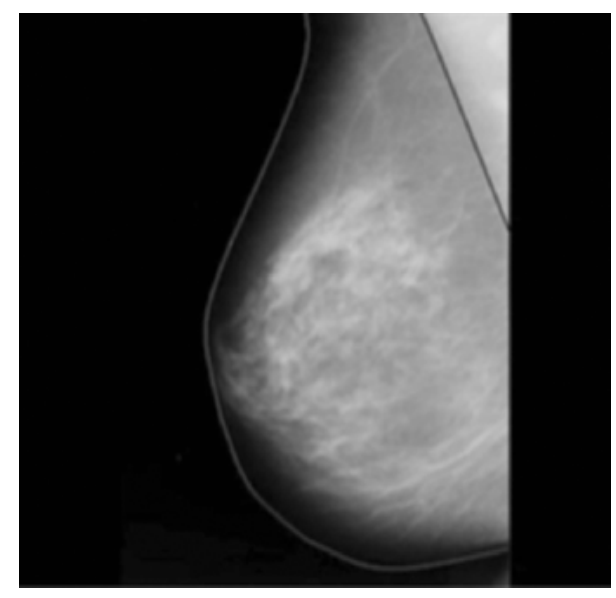

Figure 6.1: Breast anatomy of women.

\subsubsection{Breast abnormalities features}

\section{Characteristics of circumscribed masses}

Breast mass is a 3D lesion that looks more pronounced from the surrounding breast tissue. The mass appears as a dense area of different sizes and characteristics. They can be round, oval, lobular or irregular/inferred. This is shown in Figure 6.2.

Sometimes, the mass is also displayed with microcalcifications (MC). Fluid collection forms a tumor that is noncancerous. In the mammography film, it looks like a lump. The intensity of the mass tissue is a time similar to that of 


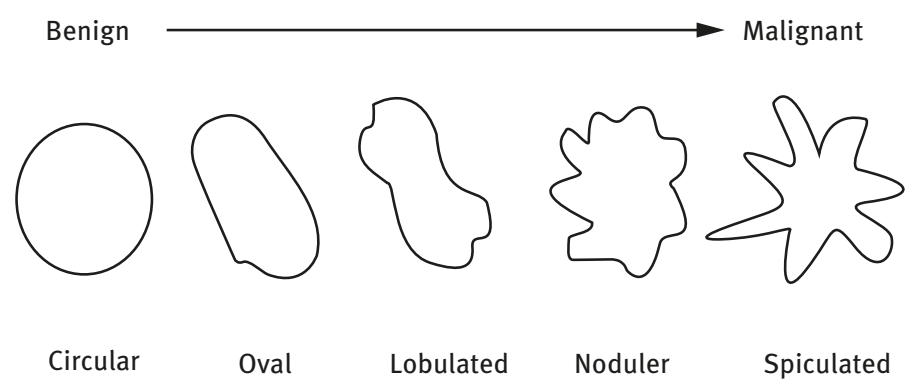

Figure 6.2: Morphologic spectrum of mammographic masses.

normal tissue. The morphology of the mass region coincided with that of the other normal tissues in the breast. This makes the job of mass detection very challenging. The radiologist estimates the probability of cancer using information about the location, size, shape, density and boundary characteristics of the mass. Features of benign lesions are well defined and compact, and are approximately circular or elliptical border. Malignant lesions are characterized by unclear and uneven boundaries. Malignant masses are sometimes surrounded by radial spiny lines.

The example of the margins associated with circular, lobular and ill-defined masses is shown in Figure 6.3.

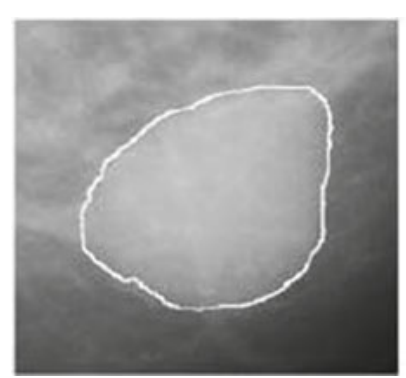

(a)

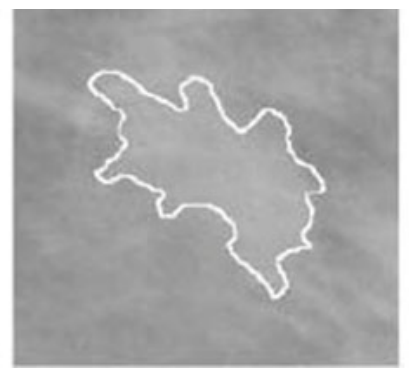

(b)

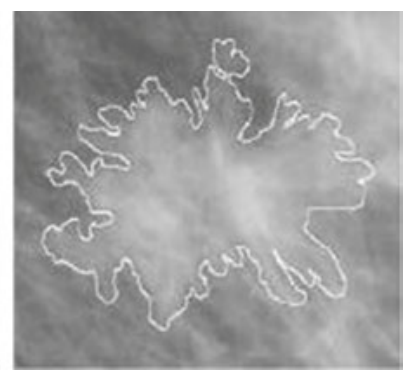

(c)

Figure 6.3: Mass examples with different shapes and borders: (a) circular shape, (b) lobular shape and (c) ill-defined shape.

The example of mammogram with malignant circumscribed mass from MIAS database is shown in Figure 6.4 .

Undefined boundaries and spiky boundaries have a high probability of malignancy. Benign processes are usually associated with the presence of a round or 


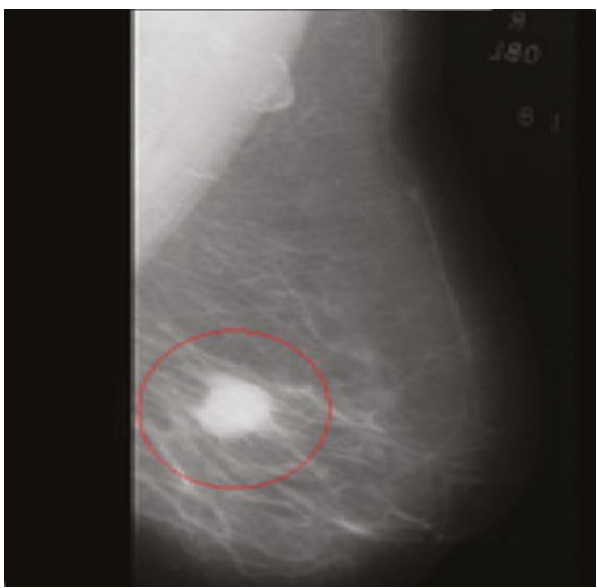

(a)

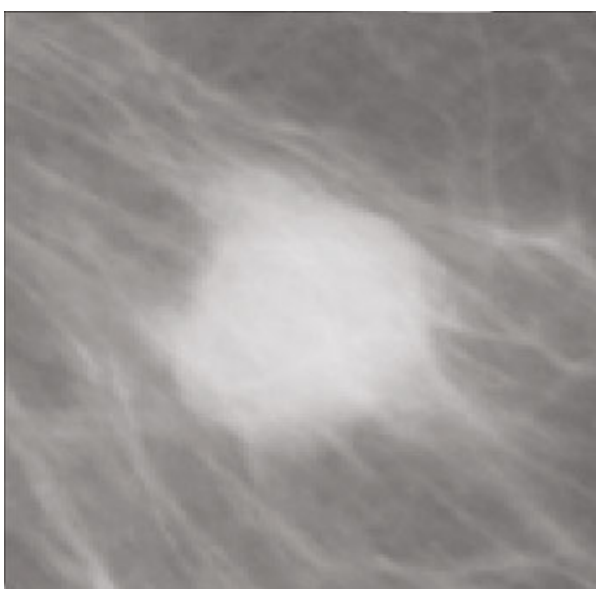

(b)

Figure 6.4: (a) Example of mammogram with circumscribed mass mdb028 and (b) zoomed encircled abnormal region.

oval mass. However, large fluctuations in the mass appearance are an obstacle to correct mammographic analysis.

\section{Characteristics of microcalcifications}

Calcification appears as a small bright spot on a relatively dark background normal tissue. These are basically small-sized calcium deposits. Important signs of malignant breast disease are the existence of calcification cluster. Malignant calcification is small and has a variable size and shape. A relatively large number of MCs exist in the cluster form. They appear as a prickly oriented branch. Benign calcifications are usually small in number, large, scattered, uniform in size and shape, and have a smooth, circular margin. They are easily visible on a mammogram. The roughness of the shape and the distribution of calcifications are used as important features for distinguishing benign and malignant calcifications. Figure 6.5 shows a mammogram with malignant MC clusters.

A careful study of the features of calcification will help the radiologist whether it is benign, which requires regular examination, and a biopsy is necessary. Size of individual calcification is not so important, but its form is determining its classification. Usually the size of the calcification is very small $(0.1-1 \mathrm{~mm})$ and is typical that the diameter is 0 . It is $3 \mathrm{~mm}$. This is a major challenge in detecting calcifications. Small calcifications may go undetected due to partial cover by the breast parenchyma. It is very difficult to find small MCs in dense background tissues. If the 


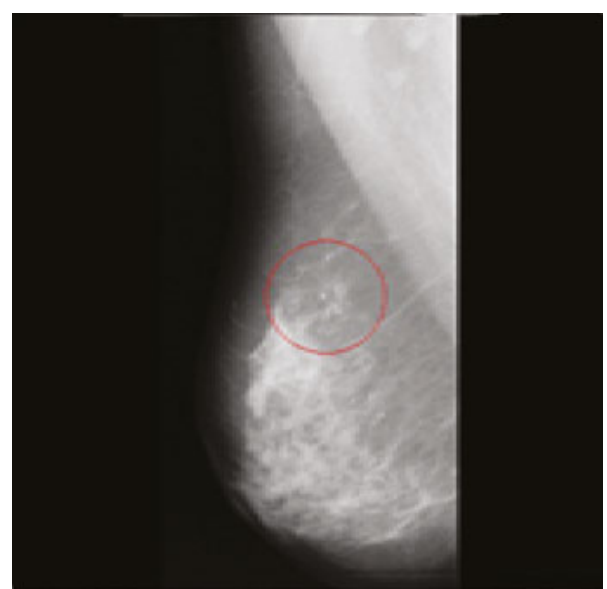

(a)

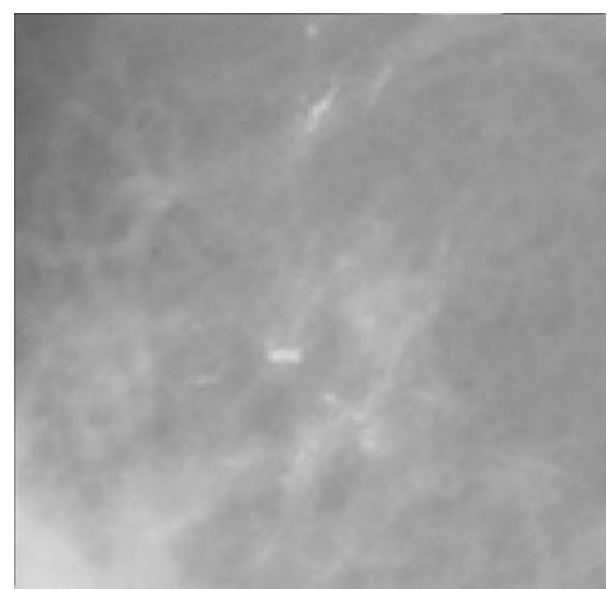

(b)

Figure 6.5: (a) Example of mammogram with microcalcification mdb249 and (b) zoomed encircled abnormal region.

background is inhomogeneous, and the calcifications have a low contrast, then the calcifications may be mistakenly regarded as noise.

\section{Characteristics of architectural distortion and spicules}

The third most common mammographic sign of cancer is architecture distortion. It strongly hints at malignant tumors. Architectural distortions are defined as a spiked deformation emanating from a point and an ordinary architectural deformation with focal bending at the edges of the real without the presence of visible masses. Figures 6.6 and 6.7 show the breast and malignant architecture distortion in the diseased area, respectively.

As one of the visible abnormalities and architectural distortion of the chest mass, asymmetry or calcification appear together, which indicates a high probability of malignancy. Injury or soft tissue damage is caused by trauma to the building distortion that is a good type. Accurate identification of architectural distortion early BC assumes an imperative job in early identification.

\subsubsection{Features used for the detection of abnormalities}

As explained in the previous section, the bounding mass of a mammogram is characterized by its shape, texture and appearance of the perimeter. MC is characterized by roughness of shape and size, homogeneous or heterogeneous tissue, the number 


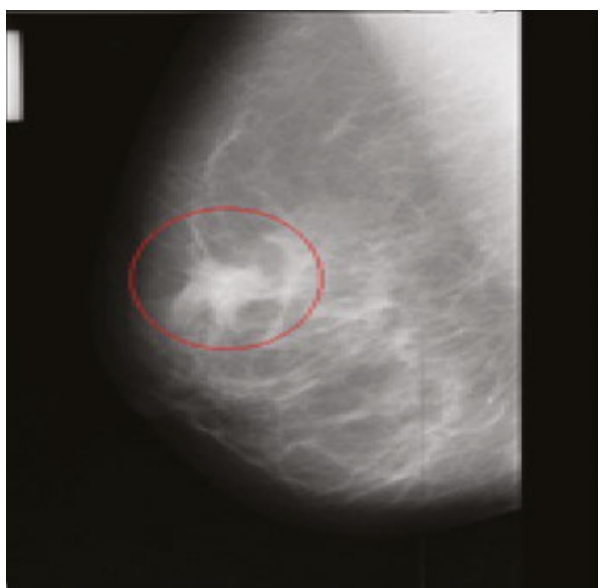

(a)

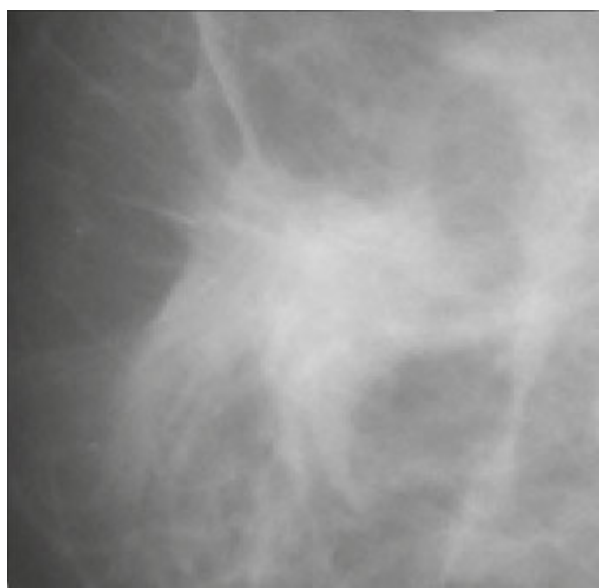

(b)

Figure 6.6: Example of mammogram with architectural distortion mdb249 and (b) zoomed encircled abnormal region.

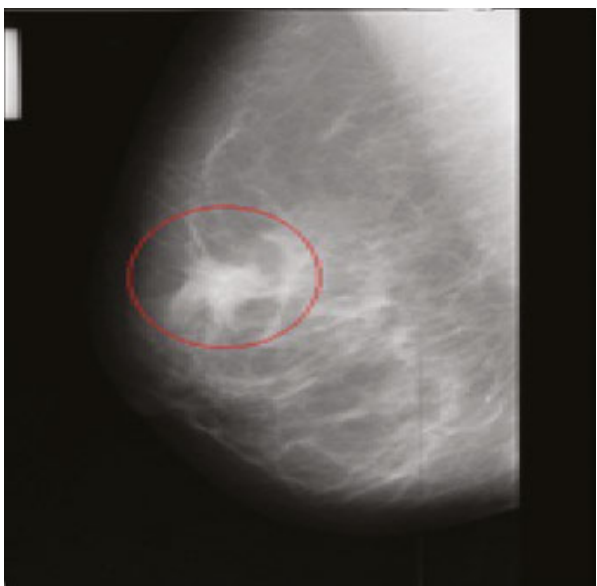

(a)

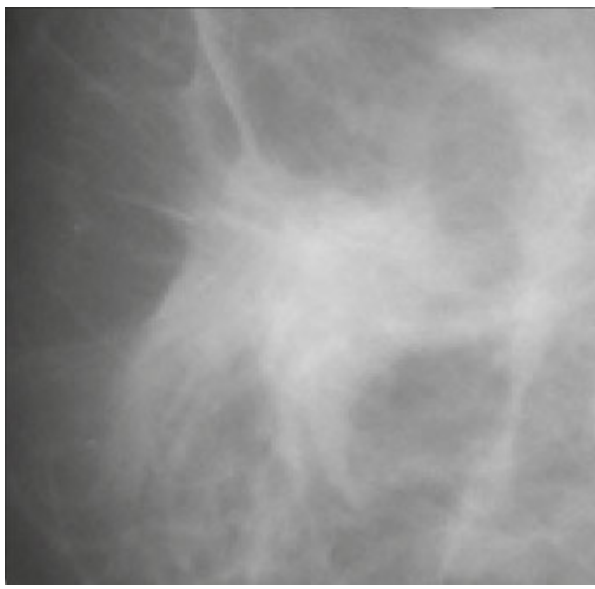

(b)

Figure 6.7: (a) Example of mammogram with spicule mdb206 and (b) zoomed encircled abnormal region.

of calcifications per unit area and so on, which is useful for detecting architectural distortions and spicule-oriented patterns. All of these functions are grouped into major shapes and features such as the texture, the features and orientation of the features. 


\subsubsection{The analysis of PCA-ICA for detection of abnormalities}

\section{Principal-Component Analysis}

The principal component analysis (PCA) is an established way to deal with statistical data analysis, feature removal and data compression. In the case of PCA, the goal is to find a small set of less redundant variables to represent the signal in much the same way as the original signal representation. In the case of independent component analysis (ICA), the goal is to determine independent components. In PCA, redundancy is estimated by connections between data elements. In ICA, decreasing the number of variables is of less importance. PCA uses only secondary statistics and is used as a preprocessing step with ICA to reduce dimensions.

PCA is popular because of its strengths, general applications and its calculations. PCA converts the initial dataset in the form of a vector into a new vector sample set that allows you to select the desired dimensions. In high-dimensional data, it is very difficult to identify patterns. PCA can be used as a powerful tool for analyzing data as it reduces its dimensions. Figure 6.8(a) indicates a plot of the two-dimensional zeromean data. Figure 6.8(b) indicates a plot of the data plotted with the eigenvectors above it.

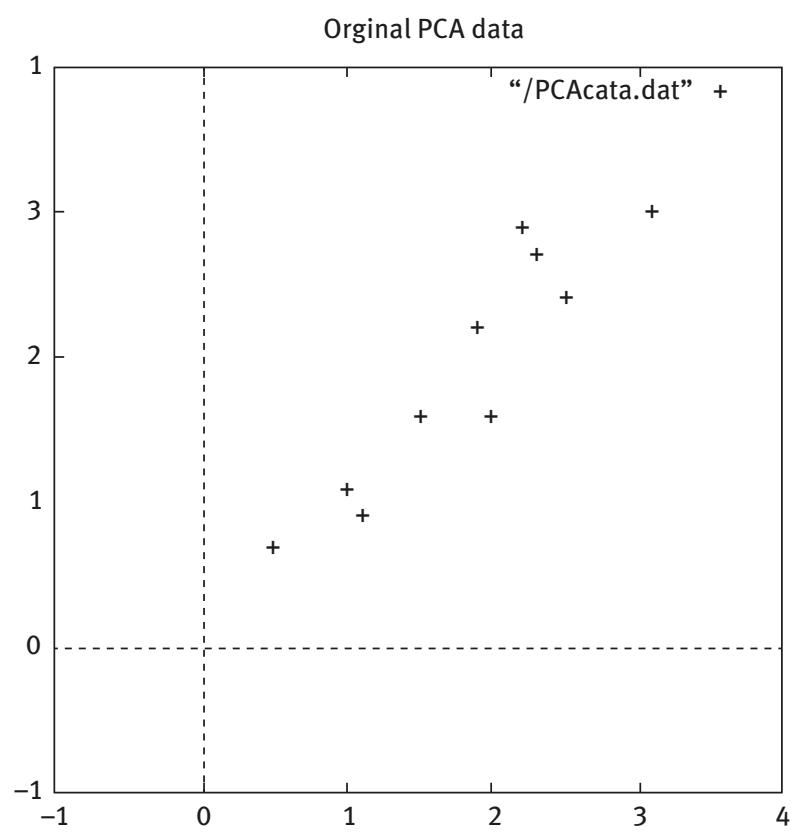

Original data on left, mean subtracted data on right side and plot of the data

Figure 6.8: (a) Plot of mean subtracted data. 


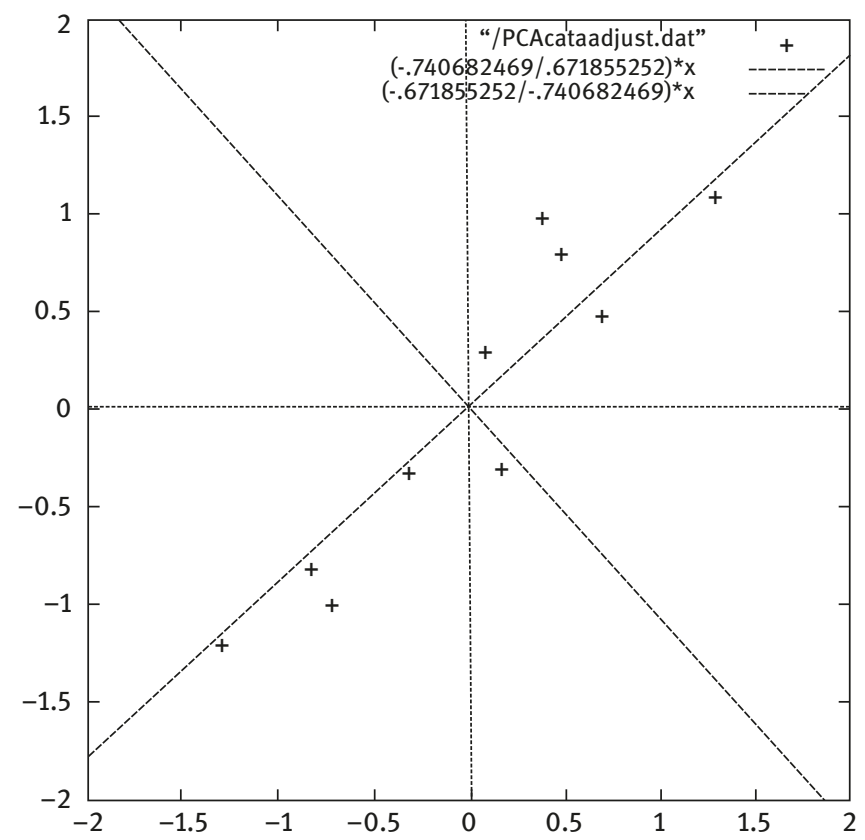

Figure 6.8: (b) mean subtracted data and eigenvectors on top.

They appear as oblique dashed lines and are perpendicular to each other, and provide important information about the patterns in your data. First, data is concentrated near the first eigenvector; second, eigenvector indicates a nonsignificant pattern in the data.

In general, images appear in large sizes. Whenever we take a picture of any type, processing, feature extraction or classification, we need to take more numbers; finally, the total size of the data matrix is significantly increased. Thus, if the size is huge, then the complexity of the calculations, time needed and the memory requirements automatically increase significantly. Hence, there is a need of dimension reduction. In an image, you can discard those redundant or less distributed features that have redundant functionality and provide less important information about the image.

PCA comes to our rescue in this situation. Suppose you have 50 images as input data. Each image is transformed into a single dimensional vector. Now all 50 images are placed as rows of a matrix and a data matrix is generated. As you can already see, the covariance of the image is calculated, and the eigenvectors and eigenvalues are given. The covariance matrix is a symmetric matrix with off-diagonal elements. The diagonal element of the covariance matrix is actually the variance of its own 
dimension. By discarding the redundant elements directly, we can obtain a new image set of reduced size with only the principal components that give the most important information from the image. Figure 6.9 shows the principal components with corresponding variance.

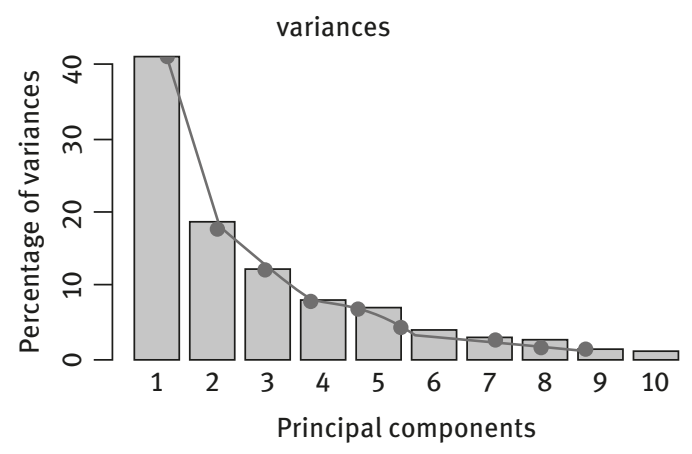

Figure 6.9: Plot of principal components with corresponding variances.

\section{Independent component analysis}

When the image is transformed from the original space region to another region, explore some of the key features of image data that are useful for image analysis. One of the simplest techniques is the main component, which is described earlier. Efficient methods for characterizing natural images are higher order statistics, but these are overlooked by the principal component. The use of independent components can overcome this limitation of the PCA. Each image can be represented as a linear combination of unnecessary independent components. Direct calculation of independent components is possible by determining the joint distribution that is not feasible. The optimization of some independence-related criteria is used to develop algorithm to determine ICA.

A statistical "latent variables" model can be used to define ICA. Vector $X$ is formed by $n$ random variables $x_{1}, x_{2}, \ldots, x_{n}$. Vector $X$ can be represented by linear combinations of $n$ random variables $s_{1}, s_{2}, \ldots, s_{n}$, which are statistically mutually independent, denoted by a random vector $s$, and then ICA is defined as

$$
X_{i}=P_{i 1} S_{1}+P_{i 2} S_{2}+\cdots+P_{i n} S_{n} \text { for all } i=1, \ldots, n
$$

where the " $p_{i j}$ " are some real coefficients. In vector-matrix form $X$ can be represented as

$$
X=P S
$$


Sometimes the columns of matrix $P$ are needed; denoting the columns of matrix $P$ by $p_{j}$ the mixing model can also be given by

$$
X=\sum_{i=1}^{n} P_{i} S_{i}
$$

ICA is based on the assumption that if components $S_{i}$ are unnecessary independent, and independent components must have unknown non-Gaussian distributions. Then, after calculating the matrix $P$, its inverse $W$ is computed, and the independent components are obtained by

$$
S=W X
$$

\subsection{Review of literature}

This section discusses the literature review in detail about the women $\mathrm{BC}$ prognosis prediction.

In biotechnology and medical field, image processing is an important task. Extracting important features of the images they used a texture-based method. First, Farzam et al. applied discrete wavelet transform on the image and approximation matrix is changed into $1 \mathrm{D}$, utilizing zigzag scanning and finally volatile signal features are removed. These features are more delicate to light and edge so that they can be different from the abnormal and normal individuals. Experimental result shows that the accuracy is better than the previous system. Limitations: it requires large number of cases [1].

Clustering classification of breast MC into malignant and benign category is a challenging task for the computerized algorithm. Alan et al. [2] used multiview classification for the classification of MC, and it is implemented using logistic regression classification. This experiment is conducted on the digital database for screening mammography and this dataset includes demographic data of the patients. Poor accuracy is its limitation:

$$
P(x)=\frac{1}{1+\exp (-(\beta 0+\beta 1 x))}
$$

where $P(x)$ is interpreted as the probability of the dependent variable, $\mathrm{X}$ is the design matrix and $\beta$ the shared parameter.

Computerized clustered MCs in mammograms suffer from the existence of FP results. Maria et al. explored the statistical estimate to determine some amount of FP that exists in the identified MC. First, they found out some amount of true positives (TP) by using Poisson-binomial probability distribution of training they used logistic regression models. Three different methods are used for MC detectors, namely context-sensitive classification model detector, support vector machine (SVM) detector 
and DoG detector. Limitations: without any $\mathrm{MC}$ it includes the number of normal cases. The huge part in a mammogram image does not have a few MCs [3].

Ting et al. used self-regulated multilayer perceptron (MLP) neural network (NN) for the classification of BC. Machine learning (ML)-NN utilizes MLP to support medical specialists in diagnosis. For categorizing the images into normal patients, benign and malignant trained ML-NN is used. Oriented ridge-like function can be expressed by only single perceptron and MLP-NN in layer-by-layer basis. But it requires high computation cost [4].

Dongdong Sun et al. [5] proposed prognosis prediction of BC techniques such as multimodal deep $\mathrm{NN}$ by combining multidimensional. In this they integrate the multidimensional data, which include copy number alternation profile, clinical data and gene expression profile. They used multidimensional data, and performance of multidimensional techniques is compared with the single dimensional data. The proposed system gives more appropriate result than the current system and it can be identified by experimental results. This method established on single source of information that has some restrictions such as an absence of noisy data, nonuniversality and singularity.

In the CAD (computer-aided detection) techniques, preprocessing of images is divided into two main steps such as removal of pectoral muscles and noise. Sreedevi et al. [6] combined ROR (robust outlyingness ratio) mechanism with the elongated nonlocal means filters that depend on the discrete cosine transform for removal and discovery of noise. For identified pictorial muscles they used a global thresholding, recognized the edge of full breast and used edge detection processes. The limitation for this strategy on a reserved database for processing, application of a straight line-based strategy is used.

Glioblastoma multiforme (GBM) is an aggressive type of brain cancer with the lowest median survival rate of patients. Chen Peng et al. [7] proposed mRMR feature selection method with multiple kernel learning classification method for the prediction of GBM prognosis. The survival rate of patients is different for every subtype of glioma. For this experiment they used the cancer genome atlas dataset of various types of cancers. They improves the prognosis prediction accuracy of GBM and compared the performance with the one kernel method using same dataset. Because of the different datasets and process, method cannot compare with other researches directly:

$$
k^{\prime}=\sum_{i=1}^{n} \beta_{i} k_{i}
$$

where $\beta$ is the vector of coefficients for each kernel, $K$ the kernels and $N$ the number of kernels.

In this advanced technology, new possibilities are coming from a scientist to gather multimodal data in the different applications such as medical imaging, brain/body machine interface, bioimaging and omics. Fabian et al. [8] provide a deep study on utility with dissimilar biological data and relative study of the deep 
learning techniques, reinforcement learning and the combination of deep learning and reinforcement learning in mining of biological data. Heavy computing memory and power desired by this method are the main limitations.

Khan et al. [9] proposed techniques designed for irregular classification of breast mass from the digitized mammography images. They considered for texture feature as a local binary pattern (LBP) and this feature is categorized by the ML techniques such as SVM. Mammography is a technique used to take multiple views and angles of the breast. They classified "mediolateral-oblique" views and "cranialcaudal" views separately and finally combine the classification results for accurate diagnosis. This technique reduces the classification error and it reaches the high reorganization rate. It contains extra local spatial information. Effectiveness of system is not good:

$$
\left[\frac{1}{n} \sum_{i=1}^{n} \max \left(0,1-y_{i}\left(w \cdot x_{i}-b\right)\right)\right]+\lambda w^{2}
$$

where $y_{i}$ is the itch target (i.e., in this case, 1 or -1$),\left(w . x_{i}-b\right)$ is the current output and $\lambda$ is the tradeoff between increasing the margin size.

Farag Alhsnony et al. [10] proposed a new automated technique and detected the region of pectoral muscle by utilizing a bit depth and edge processes system for segmenting a digital mammogram image. For more accuracy, CAD system is required. To determine the $\mathrm{BC}$, it was found that the third-order fitting curve can be used. Limitation is recognition of pectoral muscle region in mammogram image needs a series of mathematical analysis method to be recognized.

Qiao Pan et al. [11] proposed a novel NN that depends just on character-level representation for disease classification. Their model gives information through the CNN (convolutional neural network) and remaining network over characters. Output of this is given to a GridLSTM (grid long-short-term memory). GridLSTM model is used to capture both forward and backward long-term dependencies between characters. For character-based grid CNN, they need to extract thick feature vectors.

$\mathrm{BC}$ is a very dangerous disease in the United States and the United Kingdom. It is also one of the leading diseases with the highest mortality rate. This BC is an irregular growth of cells from the blood tissue and tumors can be malignant or benign. Early recognition builds the odds of survival and decreases the mortality rate. The method of classifying the mammogram based on the characteristics extracted using LBP and the LGP (local gradation pattern) with the result, and histograms are compared. LBP and LGP techniques are commonly used for finding pattern in textural analysis. The generated pattern is used to classify tumors using an SVM to classify BC [12].

$\mathrm{BC}$ is one of the important reasons for death among women. Many techniques have been developed to detect and diagnose the disease, but have not improved the results among the number of deaths caused by this disease. Therefore, early detection 
and diagnosis is the only way to prevent them from death. Previously, radiologists used to manually check the signs of cancer with mammographic images, but they did not give an effective result. Therefore, in this chapter, a new technique has been implemented for detecting and diagnosing the $\mathrm{BC}$, and for the classification of cancer, image processing techniques such as texture method and KNN method were used, and it was possible to obtain the result where the reliability was high [13].

The underlying motivation of this study to a number of various mechanisms, specifically the remodeling of collagen fibers in tumor-related stroma, is that the redesigning of collagen fibers, hematoxylin and eosin has been accounted for to be identified with the survival of patients in the tumor microenvironment. The purpose of this chapter is to classify interstitial regions with maturity level and show that this classification coincides with the classification of skilled observers, and the method in which we combined a random decision tree classifier for the categorization of BC intertissue regions, the basic image features of multiscale and the LBPs [14].

Detection of breast muscle is an important invention in improvement in diagnostic detection of $\mathrm{BC}$. The author proposes an intensity-based technique for the pectoral muscle boundary detection in the mammogram images. A $3 \times 2$ enhancement filter mask has been proposed and it is applicable for the image of thoracic region of the mammogram. Thorax boundary point was detected by the threshold method. Finally, the boundary of the pectoral muscle was obtained by connecting all the detected boundary points. The 322 mammography of the 320 digital mammography (MIAs) database was examined. The mean FP rate and the FN rate showed the accuracy of the proposed method [15].

Recognition image processing technology related to medical image is explained. There are a few assortments of proliferative malignancy that are now famous by researchers who have hit an aggregate to 100. Every single malignant growth is unique in its kind to be acclaimed along with the signs. This chapter has some expertise in more than a few image processing algorithms that are included in the diagnosis of breast melanoma, which is a risky cancer inspired in women worldwide. On measurements for this addition, learning is taken from the international body of cancer research - the WHO (World Health Organization) and the American Cancer Society. Benchmarking of current and previous studies has been shown to enhance long-term findings [16].

Breast malignant growth is the most widely recognized disease of ladies everywhere throughout the world. The most commonly used screening technique is mammography. To decrease the expense and remaining task at hand of radiologists, we used a computer-assisted approach to classify and localize calcifications and masses in mammogram images. Author improves the conventional approach and applies a deep CNN for automatic feature learning and classifier construction. In computer-aided mammography, the deep CNN classifier loses the details of an image from resizing at the input layer, so instead of directly resizing to the full mammogram image, the trained classifier labeled the image patches, and the full 
mammogram image of the work adapted to it does not have a significant anomaly. The most advanced deep CNNs are analyzed on the performance of classifying the anomalies. From the experimental results, Vgnet was $92.3 \%$ in classification. It was shown that it received the highest overall accuracy at 53\%. To localize the anomaly, ResNet is selected to calculate the class activation map [17].

In their lives, among $8 \%$ of women diagnosed with $\mathrm{BC}$, and after lung cancer, $\mathrm{BC}$ is the second most basic reason for death in both developed and underdeveloped countries. BC is characterized by gene mutations, consistent torment, change in size, color (redness), texture of the skin of the breast. BC classification indicates to the fact that pathologists locate a deliberate and target prognosis, and generally the most regular classification is binary (benign/malignant) cancer. Currently, ML technology is widely used in $\mathrm{BC}$ classification problems. They provide high labeling exactness and efficient diagnostic functions. Two dissimilar classifiers for the classification of $\mathrm{BC}$ are naive Bayes classifiers and near-knee stop classifiers. They propose the examination of two new classifiers and assess their precision utilizing cross-validation [18].

Faye et al. [19] examined the normal and abnormal tissues in mammography using the deep learning method. VGG-16CNN deep learning architecture with a convolution filter of $(3 \times 3)$ from the Irma dataset is implemented on the mammogram ROI. The deep feature matrix is the first completely linked layer. The results have been calculated using a cross-validation of binary tree, SVM, simple logistical and 10 times on $\mathrm{KNN}(k=1,3)$ classifiers. In this method, AUC1, the categorization accuracy of $100 \%$ was obtained at 0 .

In [20], CAD system, extraction of the breast region which removed the pectoral muscle and delineation of the breast contour are indispensable pretreatment processes. Predominantly, it allows the study for anomalies limited to the area of the breast without an excessive effect from the background of the mammogram. A new method for identification and removal of pectoral muscles in the mediolateral oblique (MLO) field of mammogram using the iterative threshold method is presented. The algorithm also identifies the presence of abnormal axillary lymph nodes, which are the main signs of $\mathrm{BC}$, and detects the boundaries of the breast.

Over the past few years, a few ML methods have been proposed to structure an accurate categorization system for some medical problems. In this chapter, the authors relate and analyze the classification of bosom malignant growth with various AI calculations utilizing $k$-fold cross-validation (K-CV) technology. The decision tree is a simple Bayesian classify classifier using SVM algorithm three dissimilar kernel features using NN original and predictive Wisconsin BC. A relative investigation of the study focuses on the effects of $k$ in $k$-fold cross-validation and achieves higher accurateness. The author uses the benchmark dataset from the UCI in experimental theory. It is common to choose $k=10$ for KCV. But this is due to the increase in computational costs. It is necessary to train more models. Overall results showed significant conclusions. $k$-Value of $k$ is in fold cross-validation [21]. 
The most general cause of $\mathrm{BC}$ deaths in women and the reasons for its cause are not yet fully known; however, quick detection of $\mathrm{BC}$ can be decreased with associated morbidity and death rate. Accurate removal of pectoral muscle is based on accurate detection to pectoral muscle boundaries. This suppression in grayscale mammogram images can increase the accuracy of the results of CAD techniques used in previous detection of BC. This chapter proposes a new approach based on similarity between strength to delineate pectoral muscle boundaries, using a measure of semantic similarity between words in natural language processes and features of information retrieval Fi. To achieve good results, use morphological operations to remove unnecessary element mammograms from such radiation-impermeable artifacts [22].

Digital breast tomosynthesis gives huge chances in both cancer diagnosis and detection. It provides a high-quality picture and beats some inherent limitations of traditional 2D digital mammography due to the coinciding tissues that make cancer detection difficult. Unluckily, the quality of the tomographic images is straightly correlated to the radiation dose of the patient and there is significant pain due to the parameters established by the specialist throughout the mammographic examination. Typically, the tomosynthesis breast provides a small increase in the dose of radiation with respect to the mammography $2 \mathrm{D}$. The aim of the study was to compare the performance of digital mammography and tomosynthesis, and to investigate the relationship between the dose of radiation to the patient and the quality of the image obtained [23].

Clustered MCs is the earliest symbols of BC. Moghaddam et al. [24] planned a new CAD system for the automatic detection of two-step MC. First, the pixel corresponding to the possibility of fine mineralization is found for multilayer feed forward control using NNs employing the input of the network of four wavelets, which are characterized by two gray levels. The output of the network is transformed into a probable MC object using a four-point spatial connection. Next, they remove 25 features from potential MC objects and use a variety of Adaboost SVM (DA-SVM) and three other classifiers to identify individual Mc. Free response operating characteristic curve was issued to calculate CAD system performance and one per image by utilizing the DA-SVM.90 at a cost of 043fp. The average TP detection rate of $44 \%$ was achieved, and the very good detection performance of the CAD system was shown.

A CAD system can be provided as a second view for radiologists. This chapter describes the outline of recent development in CAD technique. Anomaly detection, anomaly classification and content-based image retrieval are outlined. On the abnormality detection, the following were introduced: microcalcium detection, mass detection and multiview base detection. On the abnormal classification, the microcalcium classification and the mass classification are shown (Table 6.1) [25]. 
Table 6.1: Comparative analysis.

(a) comparative study for image preprocessing.

\begin{tabular}{|c|c|c|c|c|}
\hline $\begin{array}{l}\text { S. } \\
\text { No. }\end{array}$ & $\begin{array}{l}\text { Feature } \\
\text { extraction }\end{array}$ & Methods & Advantages & Disadvantages \\
\hline \multirow[t]{4}{*}{1.} & \multirow[t]{4}{*}{ Texture } & $\begin{array}{l}\text { Gray-level co- } \\
\text { occurrence matrix } \\
(\text { GLCM) }[26]\end{array}$ & $\begin{array}{l}\text { It describes the texture of image } \\
\text { by calculating precise pixel values } \\
\text { and then removing statistical } \\
\text { measures from this matrix. }\end{array}$ & $\begin{array}{l}\text { It takes additional } \\
\text { time. }\end{array}$ \\
\hline & & $\begin{array}{l}\text { Local binary } \\
\text { pattern (LBP) [11] } \\
\text { Local gradient } \\
\text { pattern (LGP) [11] }\end{array}$ & $\begin{array}{l}\text { Lower intensity pixels can be split } \\
\text { more accurately than LGP. } \\
\text { LGP has better accuracy. LGP has } \\
\text { smaller detection fault in } \\
\text { comparison to LBP and better } \\
\text { computational simplicity. }\end{array}$ & $\begin{array}{l}\text { Not more accurate } \\
\text { than LGP. } \\
\text { It cannot split the } \\
\text { lower intensity pixels. }\end{array}$ \\
\hline & & $\begin{array}{l}\text { Chebyshev } \\
\text { moment }\end{array}$ & $\begin{array}{l}\text { Used for distinct and orthogonal } \\
\text { moment values. }\end{array}$ & $\begin{array}{l}\text { Random variable } \\
\text { differs from its mean } \\
\text { by more than } k \\
\text { standard deviation. }\end{array}$ \\
\hline & & $\begin{array}{l}\text { LAWS measures of } \\
\text { texture }[12,27]\end{array}$ & $\begin{array}{l}\text { LAWS technique uses a } 5 \times 5 \text { mask } \\
\text { of convolution matrix to calculate } \\
\text { the texture energy. Four main } \\
\text { characteristics are level, edge, } \\
\text { spot and ripple. It takes less time. }\end{array}$ & \\
\hline \multirow[t]{3}{*}{2.} & \multirow[t]{3}{*}{ Shape } & Compactness & $\begin{array}{l}\text { For circle, compactness value is } \\
\text { zero and this value grows with } \\
\text { roughness of elongation of the } \\
\text { main object. }\end{array}$ & $\begin{array}{l}\text { For translation, } \\
\text { rotation, starting point } \\
\text { and the size of the } \\
\text { contour compactness } \\
\text { measure is invariant. }\end{array}$ \\
\hline & & Spiculation index & $\begin{array}{l}\text { Measure of the degree of thinness } \\
\text { of spicules, and has supportable } \\
\text { consumption level. }\end{array}$ & $\begin{array}{l}\text { A good performance } \\
\text { would occur when } \\
\text { there is a very high } \\
\text { level of instability. }\end{array}$ \\
\hline & & $\begin{array}{l}\text { Fourier factor/ } \\
\text { Fourier transform }\end{array}$ & $\begin{array}{l}\text { It is not difficult to appliance and } \\
\text { is based on a well-built theory of } \\
\text { Fourier analysis }\end{array}$ & $\begin{array}{l}\text { Fourier transform does } \\
\text { not supply limited } \\
\text { shape information. } \\
\text { After the Fourier } \\
\text { transform, local shape } \\
\text { information is spread } \\
\text { to all coefficients and } \\
\text { not limited in the } \\
\text { frequency area. }\end{array}$ \\
\hline
\end{tabular}


(b) comparative study for classifiers.

\begin{tabular}{|c|c|c|c|}
\hline $\begin{array}{l}\text { S. } \\
\text { no }\end{array}$ & Classifiers & Advantages & Disadvantages \\
\hline 1 & SVM [1] & $\begin{array}{l}\text { It has a regularization parameter, which makes } \\
\text { the user think about avoiding overfitting, } \\
\text { Utilize a structural risk minimization to } \\
\text { diminish error of learning machine. }\end{array}$ & $\begin{array}{l}\text { Long training time for large } \\
\text { datasets. }\end{array}$ \\
\hline 2 & Multiview [2] & $\begin{array}{l}\text { Other characteristics in both views and density } \\
\text { types are performed by curvelet rotation- } \\
\text { invariant feature. }\end{array}$ & Time consuming. \\
\hline 3 & CADx $[3,28]$ & $\begin{array}{l}\text { Information provided by model helps reader to } \\
\text { increase the accuracy for detection. }\end{array}$ & $\begin{array}{l}\text { Existence of high levels of } \\
\text { FPs affects the accuracy of } \\
\text { CADx classifier. }\end{array}$ \\
\hline 4 & ML-NN [4] & $\begin{array}{l}\text { ML-NN can categorize the input data into three } \\
\text { classes such as normal patients, benign and } \\
\text { malignant. }\end{array}$ & $\begin{array}{l}\text { It is difficult to show the } \\
\text { problem to the network. }\end{array}$ \\
\hline 5 & DNN [8] & $\begin{array}{l}\text { DNN always uses multiple features and to } \\
\text { display the outcome of model, it uses hidden } \\
\text { layers. }\end{array}$ & $\begin{array}{l}\text { Needs large dataset, typical } \\
\text { feed forward network where } \\
\text { the input flows from the } \\
\text { input layer to the output } \\
\text { layer through number of } \\
\text { hidden layers, which are } \\
\text { multiple layers. }\end{array}$ \\
\hline 6 & KNN & $\begin{array}{l}\text { For parallel implementation it is easy to work } \\
\text { with local info. }\end{array}$ & $\begin{array}{l}\text { Requires more space for } \\
\text { storage. It sometimes slows } \\
\text { in categorizing tuples. }\end{array}$ \\
\hline 7 & CNN [17] & $\begin{array}{l}\text { Mainly used for accuracy in image recognition. } \\
\text { CNNs are very good feature extractors. }\end{array}$ & High computational cost. \\
\hline
\end{tabular}

\subsection{Methodology}

\subsubsection{Multilayer perceptron}

\section{Preprocessing stage}

The mammogram images can be preprocessed to improve its quality and highlight the agency of images. Input mammograms shall conform to the appropriate resolution for training. The digital mammograms of normal patients and patients with BC are included in the training data. Medical imaging may contain undesirable noise; 
therefore, medical imaging is tortured to improve image quality. The improved images are then tagged and entered as training datasets to enter ML-NN for the purpose of training(Figure 6.10).

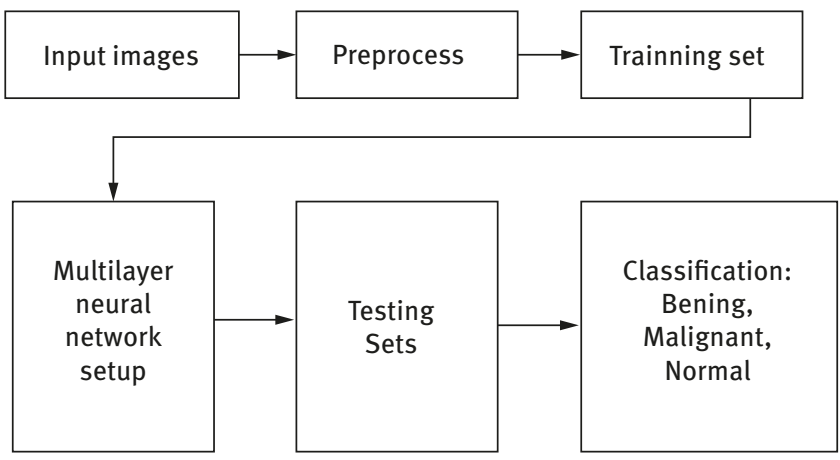

Figure 6.10: Multilayer Perceptron Neural Network for Classification of breast cancer.

\section{Multilayer perceptron}

The main objective of the this chapter is the operation of multilayer perceptron (MLP) to classify BC. The MLP consists of neurons called perceptron. In regard to the weights of neurons in the input nodes, the generation of the output by the employment of the mathematical function of activation is not linear, and the linear combination is generated by the perceptron by computing a neuron of output from multiple inputs that are valued real. The input node signal spreads through the MLP NN layer by layer.

Generally, MLP is adopted to solve problems involving supervised learning. It is indicated that a training dataset with input-output combination pairs and tags are used for input. Therefore, the MLP should be determined on the basis of training equipment.

The MLP is created with many properties like bac propagation. The flow of MLP with backpropagation is shown in Figure 6.11.

It can be used in combination of two or more phases in Figure 6.12. The black node represents the initial input. The main two stages participate in the training of this network. During the first phase, the input nodes are generated forward and the outputs of each output node are calculated. Then from expected output value each output node is subtracted. Therefore, in phase 2, all generated output node errors are passed to the opposite side, and the error weight is stable. These two phases are looped until an acceptable value is reached.

The authors propose to introduce a new technique for early prediction and comparative study of extracting the feature with and without eliminating pectoral muscle in the preprocessing phase. 


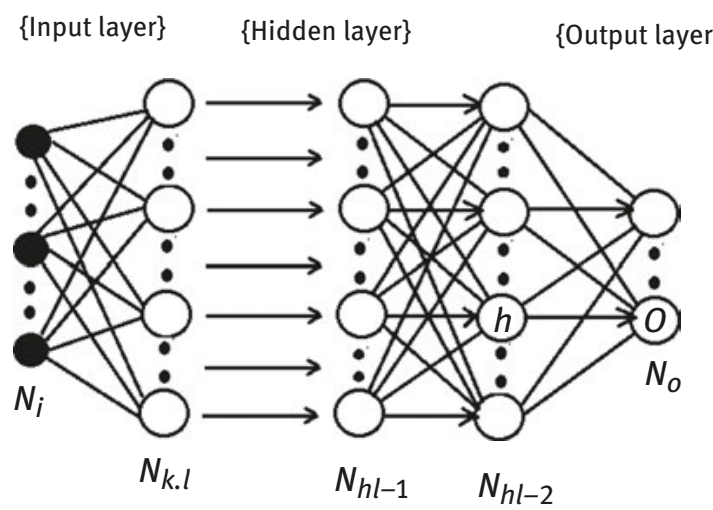

Figure 6.11: MLP with back propagation.

In the proposed system, results will be compared with the other techniques of classification. The comparison will show the accuracy and performance of system for prediction. As a result, proposed architecture should be able to predict cancer at early stage.

\subsubsection{Algorithm}

Input: $I$ = Mammogram image from MIAS database

Output: $P=$ Detection of abnormalities

Begin

Read MIAS image I from the MIAS database.

Calculate number of rows and columns in the MIAS image

Apply GLCM algorithm. It describes the texture of image by calculating precise pixel

values and then removing statistical measures from this matrix.

After that we perform LBP algorithm.

Detect the edges using Fourier Factor/ Fourier Transform.

Detect the Growing part in the image

Find out the shape of growing part calculates the area of Growing part.

Detect the type of abnormalities

End.

\subsubsection{Dataset used}

Two databases are used to carry out the proposed research work: a digitized database of the mammographic image analysis society (MIAS) and a full-field digital nonpublic. 


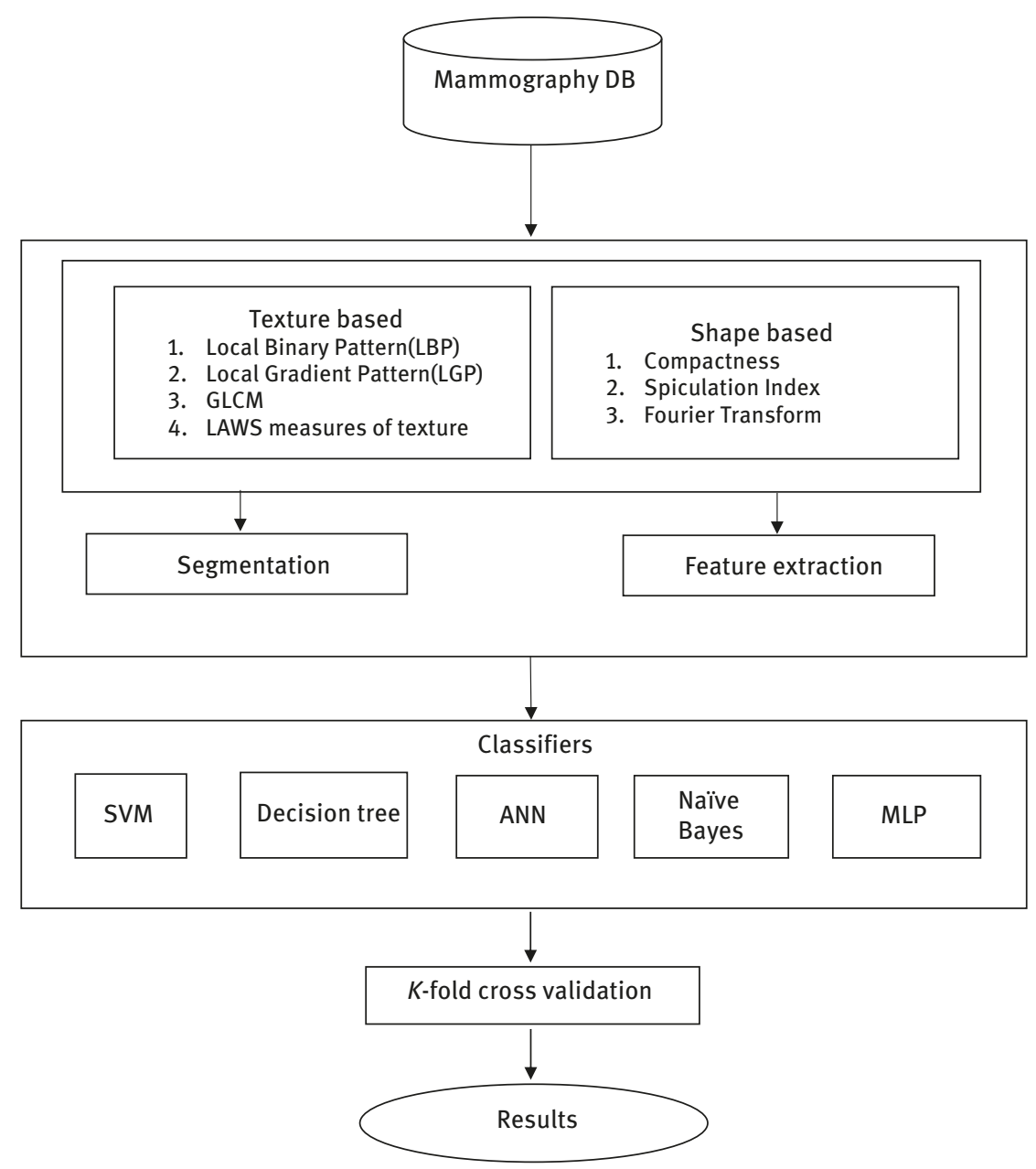

Figure 6.12: System architecture.

\section{MIAS database}

Two databases are used to carry out the proposed research work. One of it is a digitized database of the Mammographic Image Analysis Society (MIAS) and the other is Full field digital non public.

\section{MIAS dataset}

Mammograms from this dataset are caught in the MLO view and scanned at a resolution of $0.05 \mathrm{~mm}$ pixel size with a thickness determination of 8 bits. The size of 
each image is 1,024 pixels. There are a total of 322 digitized images from the UK Breast Screening Program. The 322 images contain data of 161 patients in the MIAS dataset [27]. Therefore, the MIAS dataset consists of 322 mammogram images that are divided between 208 normal patients, 66 benign and 48 malignant. These images are carefully diagnosed to identify the type and position of the abnormality. For every image in the MIAS dataset, type of background tissue, type of irregularity, center and radius of irregularity are supplied [29].

This database from the normal image with a sample anomaly is shown in Figures 6.13-6.16. For a normal breast, there are three types of breast arrangements: Fatty, fatty glandular and dense glandular (Figure 6.13). The mammogram is always captured for the breasts of both the left and right. Therefore, it is a pair of all types of mammograms. The oncologist first looks for the asymmetry of the left and right breast, and if there is asymmetry, then it is analyzed further. Figure 6.14 shows examples of benign patients and malignant patients circumscribed and other masses.

Figure 6.15 shows examples of benign and malignant patients' architectural distortion spiculations. Benign and malignant MCs are shown in Figure 6.16. In all these images, there is an anomaly in either the left or the right breast. Abnormalities can easily be seen on a fat background, whereas if the breast composition is dense, the abnormality is not visible. This can be clarified from the mammogram mdb032 from Figure 6.14(b) and mdb239.3.22 from Figure 6.16. In mdb032, other clusters exist, and in mdb239, there are MC clusters that are not clearly visible due to the dense breast background.

Some preprocessing steps have been completed successfully on MIAS dataset. Figure 6.17 contains original dataset images. On that data our novel approach, that is, LBP method, helps convert it to binary format that is 0 (black) or 1 (white). It is shown in Figure 6.18. Second method is applied, that is, Laplacian filter/Laplace transform in Figure 6.19, which is used to find areas of rapid changes in edge of images. Next GLCM (gray-level co-occurrence matrix) method is used [30]. It shows features like entropy, correlation, variance and angular moment. Hence, both LBP and GLCM methods are finding texture features of images.

\subsection{Conclusion}

This chapter discussed about the early diagnosis of $\mathrm{BC}$ and the preprocessing of image and its importance. The main aim is to develop techniques that are helpful for early detection and a comparative study of extracting the feature with and without eliminating the pectoral muscle in preprocessing phase using a new method. The main contribution is to perform strong preprocessing and powerful feature extraction and selection before classification. Some abnormalities were found, such 


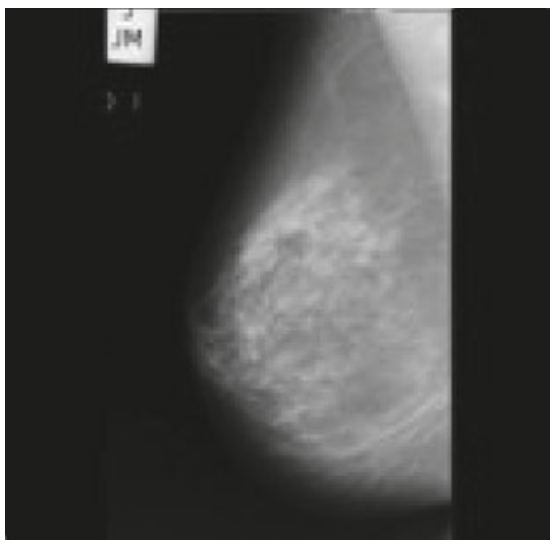

mdb003

(a)

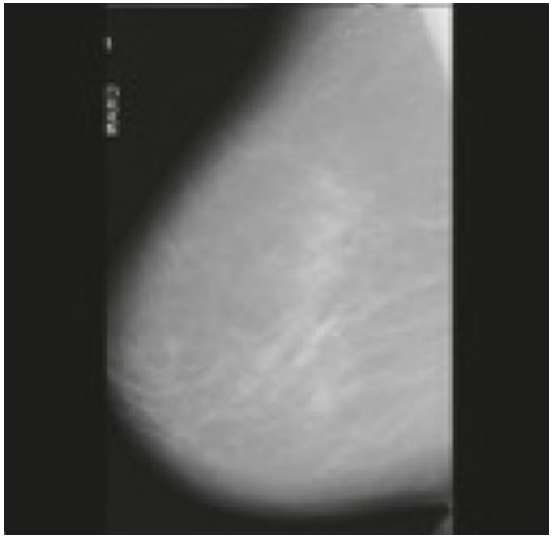

(b)

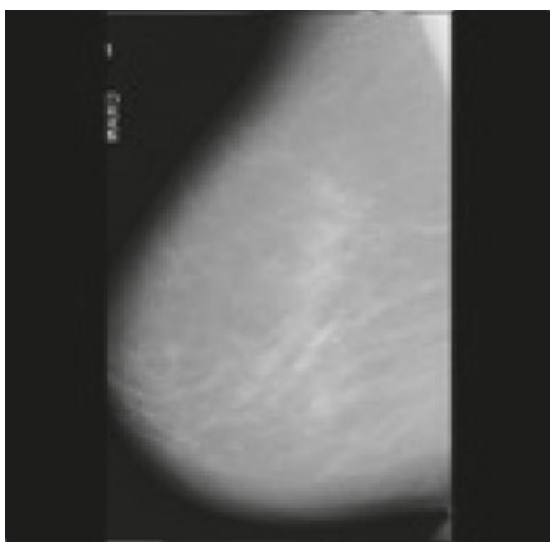

(c)

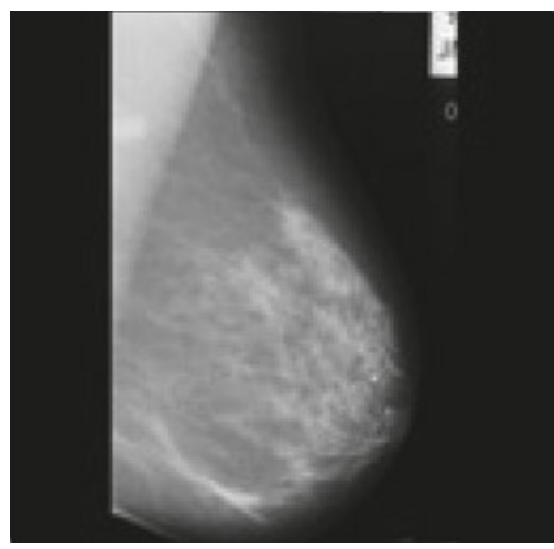

mdb004

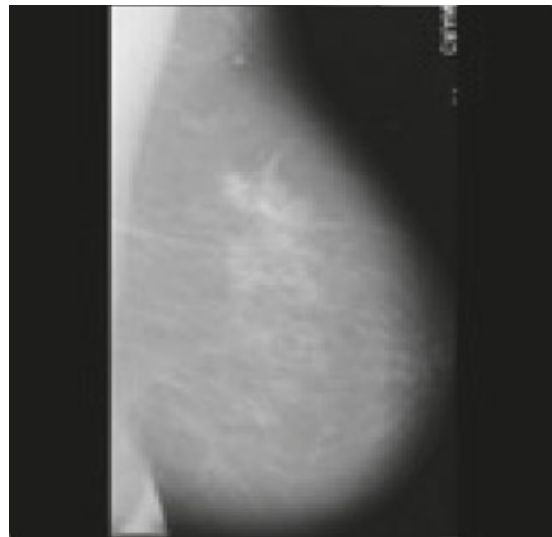

mdb008

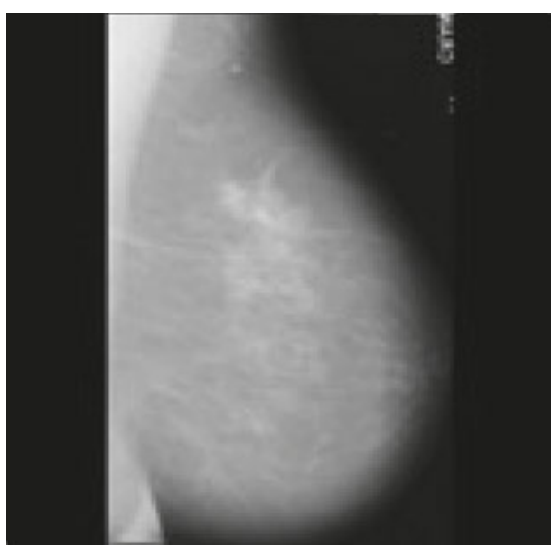

mdb174

Figure 6.13: Sample normal images from MIAS dataset [27]: (a) fatty dense; (b) fatty glandular; and (c) fatty.. 


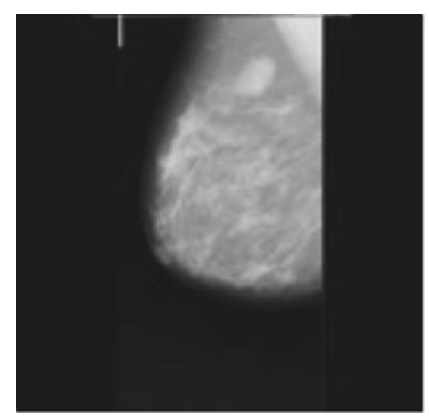

mdb015 (Benign)

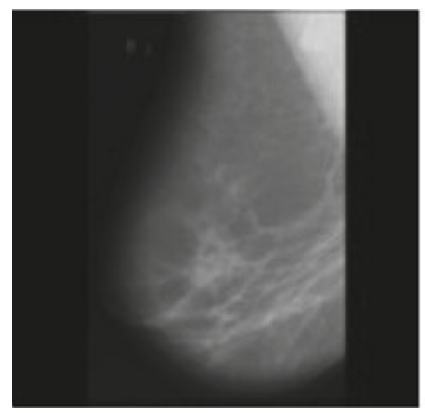

mdb027

(a)

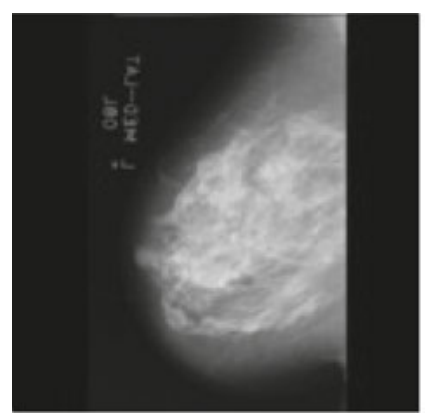

mdb031

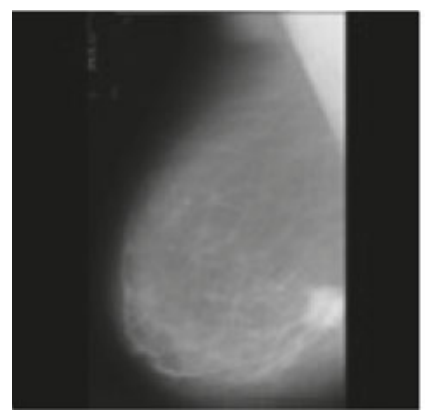

mdb271 (Malignant)

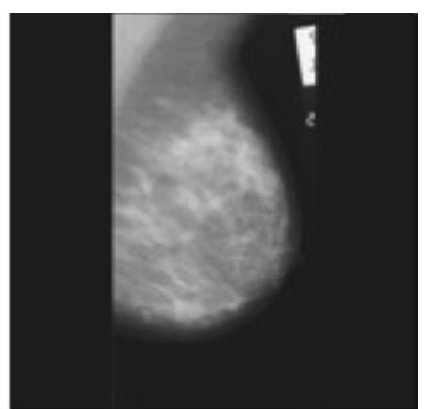

mdb015

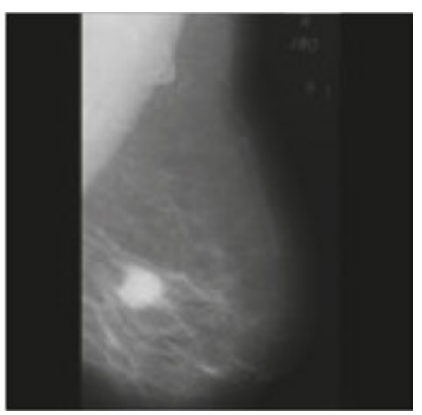

mdb028 (Malignant)

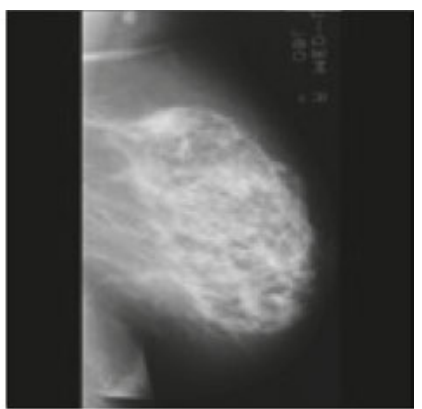

mdb032 (Benign)

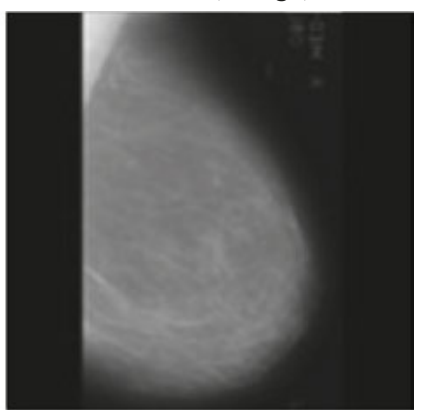

$\mathrm{mdb} 272$

(b)

Figure 6.14: Circumscribed (a) and miscellaneous masses (b) from MIAS database. 


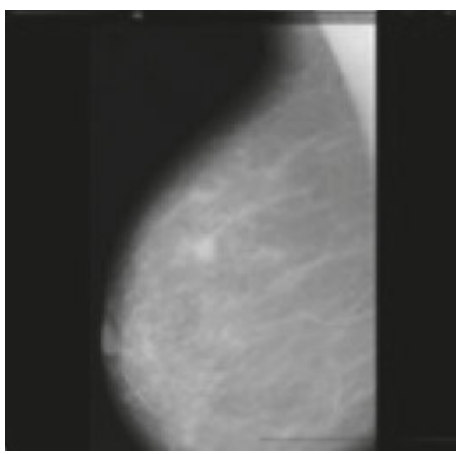

(a)

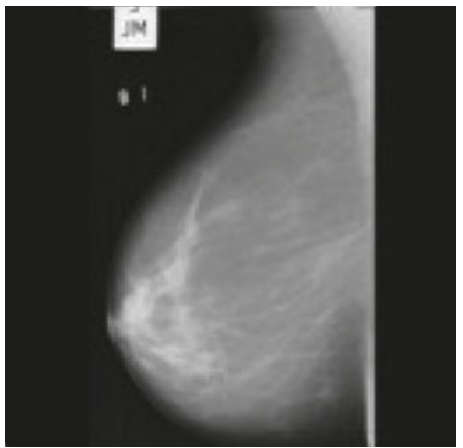

mdb195 (Benign)

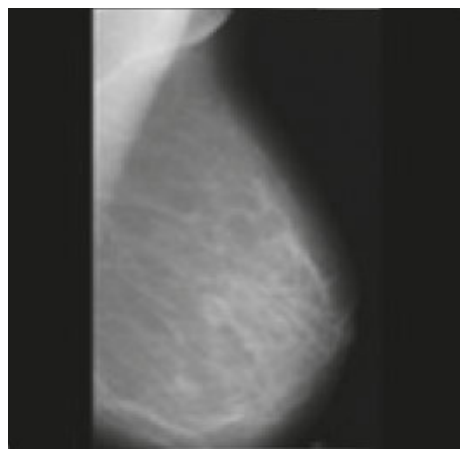

mdb 156

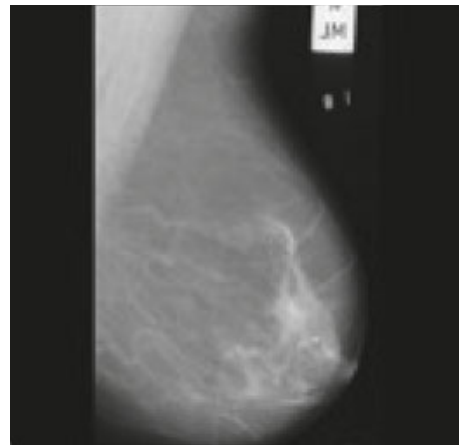

mdb196

(b)

Figure 6.15: Architectural distortion (a) and spiculations (b) from MIAS database.

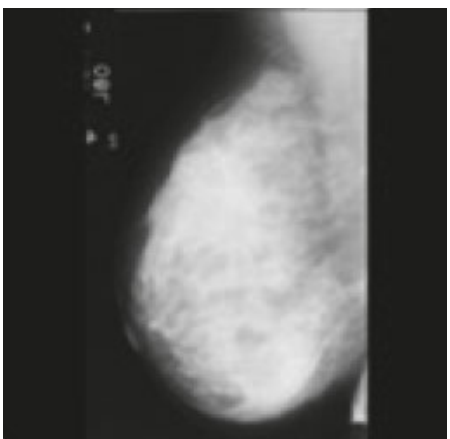

mdb239 (Malignant)

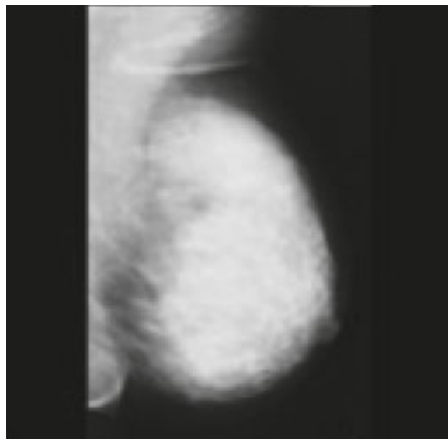

$\operatorname{mdb} 240$

Figure 6.16: Microcalcifications from MIAS database.

as identification of masses like circumscribed and ill defined, identification of MCs and identification of spiculated lesions and architectural distortions. In that 


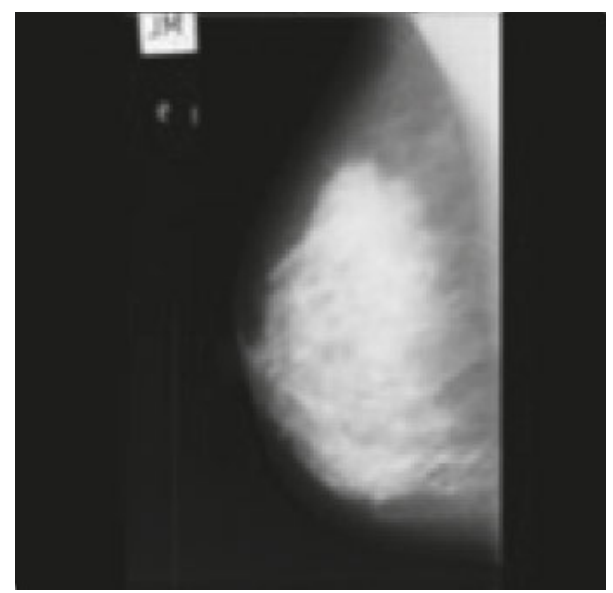

mdb003

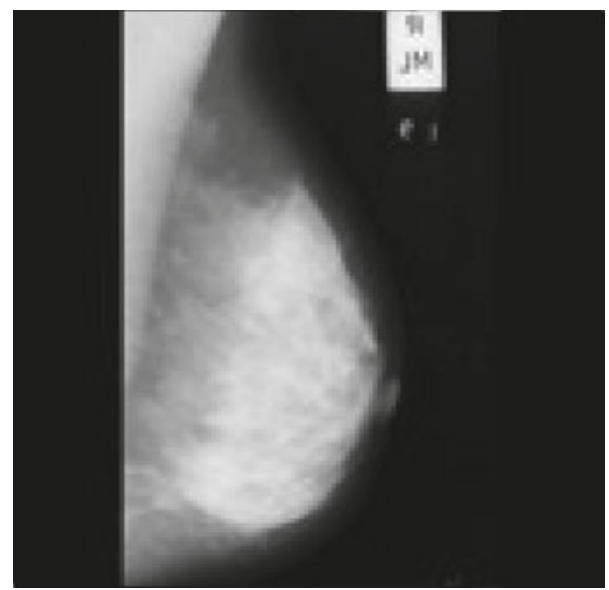

mdb004

Figure 6.17: Original data.

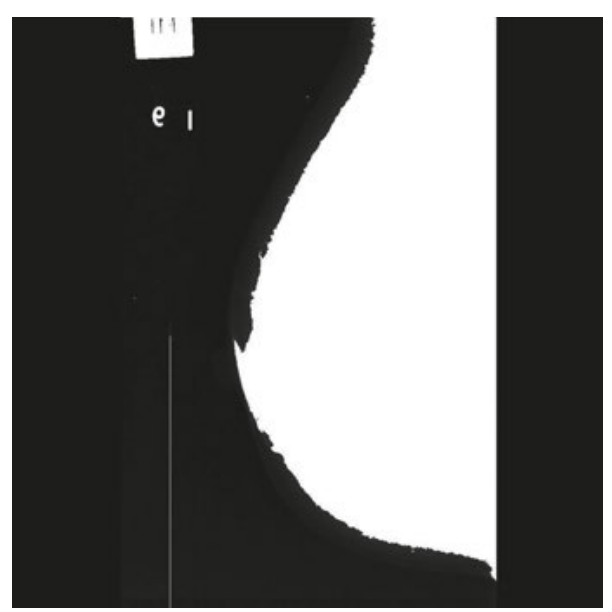

mdb003
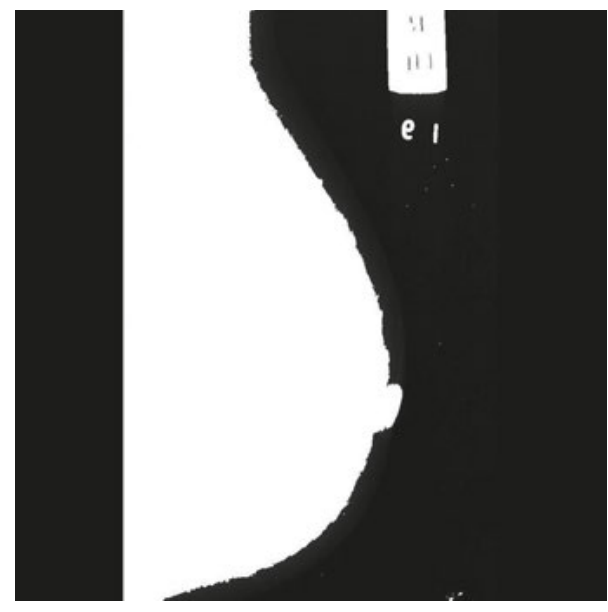

mdb004

Figure 6.18: Performing LBP.

clusters of MC is useful for early detection. For detection of abnormalities, statistical data analysis and feature extraction, ICA is used.

From comparison it was found that for texture feature, LAWS measures of texture, and for shape, compactness methods are best. MLP with recurrent NN provides more accuracy when compared with other classifiers. The proposed model is expected to predict the cancer at early stage, whether it is benign, malignant or 


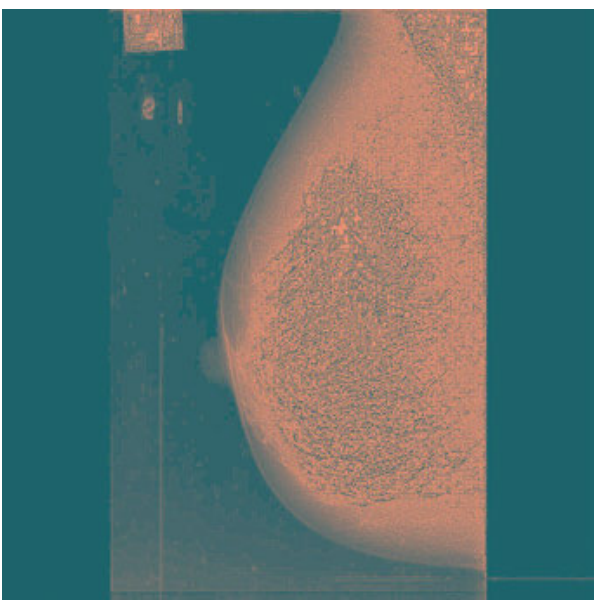

mdb003

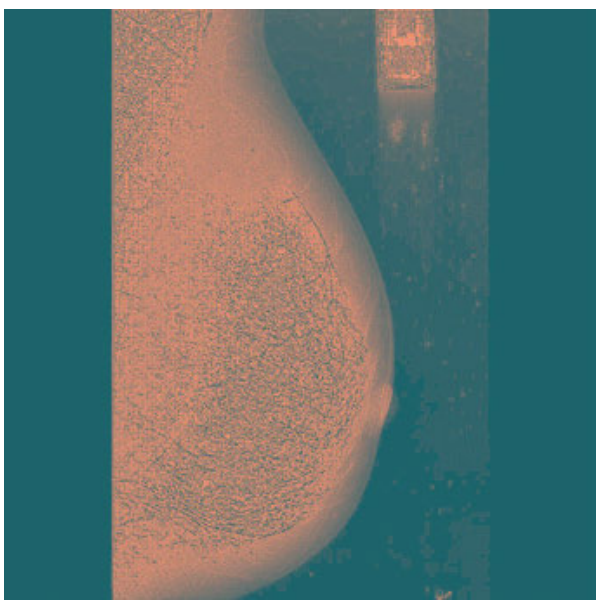

mdb004

Figure 6.19: Laplace transform.

normal. Results can be improved without the removal of pectoral muscle from mammograms that minimize the computation.

\section{References}

[1] Nezhadian, F. K., and Rashidi, Saeid. "Breast cancer detection without removal pectoral muscle by extraction turn counts feature", Artificial Intelligence and Signal Processing (AISP), 2017.

[2] Bekker, A. J., Shalhon, M., Greenspan, H. and Goldberger, J., “Multi-view probabilistic classification of breast microcalcification," IEEE Transaction on Medical Imagine, 2015.

[3] Yang, Y., Nishikawa, R. M. and Cea, S. de. "Estimating the accuracy level among individual detections in clustered microcalcifications", IEEE Transaction on Medical Imaging, May 2017.

[4] Sim, K. S. and Ting, F. F. "Self-regulated multilayer perceptron neural network for breast cancer classification", IEEE International Conference on robotics, automation and science (ICORAS)-27-19 November 2017.

[5] Sun, Dongdong, Wangand, Minghui and Li, Ao. "A Multimodal Deep Neural Network for Human Breast Cancer Prognosis Prediction by Integrating Multi-Dimentional Data”, IEEE Transaction on Computational Biology and Bioinformatics, 15 Feb 2018.

[6] Sherly, E. and Sreedevi, S., "A Novel approach for removal of pectoral muscles in digital mammogram", Elsevier, 46, 2015.

[7] Ao. Li, Chen, Peng and Zhang, Ya. "Improve glioblastoma multi-forme prognosis prediction by using feature selection and multiple kernels learning", IEEE/ACM transactions on computational biology and bioinformatics, 07 April 2016.

[8] Romero, E., Tarquino, J. and Naraez, F. “Applications of deep learning and reinforcement learning to biological data”, IEEE Transactions on Neural Networks and Learning Systems, 2018. 
[9] A1, A. Helal, K. I. Ahmed, H. A. Khan and Mostafa, R. "Abnormal mass classification in breast mammography using rotation invariant LBP”, 2017.

[10] Farag H. Alhsnony, Abdolrasol, Maher G. M., and Abadelrsool, Samei G. M. "Autoidentification of pectoral muscle region in digital mammogram images", International Journal of e-Education, e-Business, e-Management and e-Learning, February 2014.

[11] Pan, Yuanyuan Zhang, Chen, Dehua, and Guangwei Xu. "Character-based convolutional grid neural network for breast cancer classification”, IEEE 2017 International Conference on Green Informatics, 15-17 Aug, 2017.

[12] Ponraj, Narain, and Mercy, Poongodi Merlin, "Texture analysis of mammogram for the detection of breast cancer using LBP and LGP: A comparison”, IEEE Eighth International Conference on Advanced Computing, 2016.

[13] Dcruz, Michelle, and Dr. Sarode, Tanuja. "Feature exraction in mammograms using NSCT and LAWS texture analysis approach", International Journal of Engineering Research and Application, 7(8), (Part-6) August 2017, pp. 2248-9622.

[14] Reis, Sara, Gazinska, Patrycja, Hipwell, John H., Mertzanidou, Thomy, Naidoo, Kalnisha, Williams, Norman, Pinder, Sarah, and Hawkes, David J. "Automated classification of breast cancer stroma maturity from histological images", IEEE transaction on biomedical engineering, 2016.

[15] Vikhe, P. S., and Thool, V. R. "Intensity based automatic boundary identification of pectoral muscle in mammograms, ELSEVIER, 7th International Conference on Communication, Computing and Virtualization, 2016.

[16] Saranyaraj, D., and Manikandan, M., "Medical image processing to detect breast cancer - A cognitive-based investigation", IEEE 4th International Conference on Signal Processing, Communications and Networking (ICSCN -2017), March 16-18, 2017.

[17] Pengcheng, Xi, Goubran, Rafik and Shu, Chang. "Abnormality detection in mammography using deep convolutional neural networks”, arXiv:1803.01906v1 [cs.CV] 5 Mar 2018.

[18] Meriem, AMRANE, Saliha, OUKID, Ikram, GAGAOUA and Tolga, ENSARI "Breast cancer classification using machine learning", IEEE, 2018.

[19] Faye, Ibrahima, Awais, Muhammad, Meriaudeau, Fabrice and Jamal, Syed and Gardezi, Safdar. "Mammogram classification using deep learning features", IEEE, 2017.

[20] Hassan, Shayma'a A., Gouhar, Ghada K., Mohammed, S. Sayed and Farag, Fathi. "Pectoral muscle identification in mammograms for computer aided diagnosis of breast cancer”, CIBEC 2012.

[21] Nematzadeh, Zahra, Selamat, Ali and Ibrahim, Roliana. "Comparative studies on breast cancer classifications with K-fold cross validations using machine learning techniques", IEEE, 2015.

[22] Chaima Derouiche and Akram Boukhamla, "Pectoral muscle boundary detection using digital mammograms" 2014.

[23] Andria, G., Nisio, A. D., Lanzolla, A. M. L., and Attivissimo, F., Spadavecchia, M. "Image quality evaluation of breast tomosynthesis," IEEE, 2016.

[24] Moghaddam, H. A., Harirchi, F., Radparvar, P., Dehghan, F., and Giti, M.. "Two-level algorithm for mcs detection in mammograms using diverseadaboost-svm," International Conference on Pattern Recognition, 2010.

[25] Cao, L., Li, Y., Chen, H. and Ma, J., "A survey of computer-aided detection of breast cancer with mammography," Journal Health Med Informat, 2016.

[26] Cheriguene, S., Azizi, N., Zemmal, N., Dey, N., Djellali, H., and Farah, N. "Optimized tumor breast cancer classification using combining random subspace and static classifiers selection paradigms.", In Applications of intelligent optimization in biology and medicine, Springer, 2016. 
[27] Virmani, J., Dey, N., and Kumar, V. " PCA-PNN and PCA-SVM based CAD systems for breast density classification", In Applications of intelligent optimization in biology and medicine, Cham : Springer, 2016.

[28] Bhattacherjee, A., Roy, S., Paul, S., Roy, P., Kausar, N., and Dey, N., "Classification approach for breast cancer detection using back propagation neural network: a study." In Biomedical image analysis and mining techniques for improved health outcomes, IGI Global, 2016.

[29] Baker, Abu, Ayman A., Qahwaji, R.S, Aqel, Musbah J., Al-Osta, Hussam, and Saleh, Mohmmad H.. "Efficient Pre-processing of USF and MIAS Mammogram Images", Journal of Computer Science, 2007.

[30] Zemmal, N., Azizi, N., Dey, N., and Sellami, M., "Adaptive semi supervised support vector machine semi supervised learning with features cooperation for breast cancer classification." Journal of Medical Imaging and Health Informatics, 6(1), 2016. 